\title{
BMJ Stakeholder experiences with general Open practice pharmacist services: a qualitative study
}

\author{
Edwin C K Tan, ${ }^{1}$ Kay Stewart, ${ }^{1}$ Rohan A Elliott,,${ }^{1,2}$ Johnson George ${ }^{1}$
}

To cite: Tan ECK, Stewart K, Elliott RA, et al. Stakeholder experiences with general practice pharmacist services: a qualitative study. BMJ Open 2013;3:e003214.

doi:10.1136/bmjopen-2013003214

- Prepublication history and additional material for this paper is available online. To view these files please visit the journal online (http://dx.doi.org/10.1136/ bmjopen-2013-003214).

Received 13 May 2013 Revised 10 July 2013 Accepted 31 July 2013
${ }^{1}$ Faculty of Pharmacy and Pharmaceutical Sciences, Centre for Medicine Use and Safety, Monash University, Parkville, Victoria, Australia 2Department of Pharmacy, Austin Health, Heidelberg, Victoria, Australia

\section{Correspondence to} Dr Johnson George; johnson.george@monash.edu

\section{ABSTRACT \\ Objectives: To explore general practice staff, pharmacist and patient experiences with pharmacist services in Australian general practice clinics within the Pharmacists in Practice Study. \\ Design: Qualitative study. \\ Setting: Two general practice clinics in Melbourne, Australia, in which pharmacists provided medication reviews, patient and staff education, medicines information and quality assurance services over a 6-month period.}

Participants: Patients, practice staff and pharmacists.

Method: Semi-structured telephone interviews with patients, focus groups with practice staff and semistructured interviews and periodic narrative reports with practice pharmacists. Data were analysed thematically and theoretical frameworks used to explain the findings.

Results: 34 participants were recruited: 18 patients, 14 practice staff (9 general practitioners, 4 practice nurses, 1 practice manager) and 2 practice pharmacists. Five main themes emerged: environment; professional relationships and integration; pharmacist attributes; staff and patient benefits and logistical challenges. Participants reported that colocation and the interdisciplinary environment of general practice enabled better communication and collaboration compared to traditional community and consultant pharmacy services. Participants felt that pharmacists needed to possess certain attributes to ensure successful integration, including being personable and proactive. Attitudinal, professional and logistical barriers were identified but were able to be overcome. The findings were explained using D'Amour's structuration model of collaboration and Roger's diffusion of innovation theory.

Conclusions: This is the first qualitative study to explore the experiences of general practice staff, pharmacists and patients on their interactions within the Australian general practice environment. Participants were receptive of colocated pharmacist services, and various barriers and facilitators to integration were identified. Future research should investigate the feasibility and sustainability of general practice pharmacist roles.

\section{BACKGROUND}

Pharmacists are increasingly becoming part of general or family practice clinic teams. ${ }^{1}$

\section{ARTICLE SUMMARY}

Article focus

- Exploration of general practice staff, pharmacist and patient experiences with practice pharmacist services in Australian general practice clinics.

Key messages

- Integration was facilitated by colocation, communication and positive pharmacist characteristics, including credibility, adaptability and proactivity.

- Strong leadership, shared goals and the creation of benefits for patients and staff were imperative for successful implementation of pharmacist services.

- Logistical issues, especially time and adequate office space, were barriers to integration of a pharmacist into the clinics, but they were capable of being overcome.

Strengths and limitations of this study

- The study involved a private general practice and a community health clinic, representing the two main models of primary care practice in Australia.

- The study used multiple qualitative methods and recruited practice staff, patients and pharmacists, providing a rich exploration of stakeholders' experiences.

- The clinics and participants may not be representative of the general population because the practices had established interdisciplinary teams.

Integration of pharmacists into colocated primary care teams has resulted in improved medication, health and economic outcomes. ${ }^{2-4}$ In Australia, the colocation of pharmacists within general practice is rare, ${ }^{5}$ although such a role has been suggested. ${ }^{6}$ Given the prevalence of medication-related problems in general practice patients, ${ }^{7}$ the integration of pharmacists into the general practice team warrants further exploration.

Several studies have explored the opinions of general practitioners (GPs), pharmacists and patients about their interactions with pharmacists in primary care clinics, ${ }^{8-13}$ but in Australia such evaluation is sparse, given the novelty of this role. Recently, Australian stakeholders' views about integration have begun to 
emerge $;^{14-16}$ however, these studies mostly explored the opinions of stakeholders who had not experienced a practice pharmacist. The opinions expressed in these studies may thus be based on personal assumptions and theory rather than actual experience. Hence, knowledge of firsthand stakeholder experiences with actual integration of pharmacists in Australian general practice is warranted.

The Pharmacists in Practice Study (PIPS) ${ }^{17}$ was a prospective, prepost intervention study investigating the integration of pharmacists into general practice clinics in Australia. This paper describes a qualitative evaluation of the PIPS, the aim of which was to explore general practice staff, pharmacist and patient experiences with pharmacist services provided in general practice clinics.

\section{METHODS}

The PIPS methodology and the practice pharmacist role have been described previously. ${ }^{17}$ Briefly, pharmacists were located in two general practice clinics-one private practice and one community health centre-in Melbourne, Australia. Privately run general practice clinics and community health centres are the two main models of primary care medical practice in Australia. In private clinics, GPs are paid on a fee-for-service basis and patients may have to contribute copayments. Community health centres are government funded and offer a range of community health services to local residents, with a focus on health promotion and disease prevention and management. GPs are predominantly salaried and fees are charged for services according to the client's ability to pay.

The PIPS pharmacists provided short-patient and longpatient consultations, drug information and quality assurance activities on a part-time basis (approximately $8 \mathrm{~h} /$ week) over 6 months (January-July 2012). Long-patient consultations involved pharmacists performing a comprehensive medication review, on referral from the GP, usually in the clinic but sometimes in the patient's home. Pharmacists had full access to patient medical records and could discuss issues with the GP before and/or after the patient consultation. Short-patient consultations were briefer appointments where the pharmacists provided medicines information or education on specific patient needs. Drug information services included a regular medicines newsletter and answering questions from clinic staff. Quality assurance activities included a drug use evaluation programme addressing osteoporosis pharmacotherapy, a topic selected in consultation with clinic staff.

Experiences of general practice staff, practice pharmacists and patients were explored using several qualitative methods

- Semistructured telephone interviews (patients);

- Focus groups (practice staff);

- Semistructured interviews (practice pharmacists);

- Periodic narrative reports (practice pharmacists).

\section{Recruitment and data collection}

\section{Patients}

A purposive sample of patients, reflecting a range of demographic and therapeutic characteristics, who had a long-patient consultation with the practice pharmacist were approached. Semistructured telephone interviews were conducted by one investigator (ET) within 2 weeks of the pharmacist consultation. Individual interviews were used because discussions could involve personal or sensitive information about the patient's health or medicines. These were conducted by telephone for the participant's convenience. A topic guide was used to facilitate discussion (see online supplementary file 1). Recruitment continued until data saturation was reached.

\section{Practice staff}

One focus group with practice staff was conducted at each clinic during lunch breaks at the end of the PIPS. All practice staff who had worked with the practice pharmacist during the study period were invited. Focus groups were chosen in order to gain a multidisciplinary perspective by stimulation of group discussion, as well as being logistically convenient for participants. Focus groups were moderated by one investigator (ET) who facilitated the discussion using a topic guide (see online supplementary file 2); a note-taker and an observer were also present.

\section{Practice pharmacists}

Practice pharmacists participated in individual, semistructured interviews at study end, at a mutually convenient time and place (see online supplementary file 3). Narrative reports were also completed prospectively by the pharmacists at 1, 2, 4 and 6 months. A set of reflective questions adapted from a previous study ${ }^{18}$ was provided to the pharmacists to assess processes, functions and personal experiences (see online supplementary file 4). Periodic narrative reports were used as they enabled the prospective capture of experiences and issues encountered by pharmacists during the establishment of the service, rather than relying on recall at the end of the study, thus allowing the researchers to observe the pharmacists' progression and development throughout the study.

Interview and focus group guides were developed by the research team based on the literature and the nature of the practice pharmacist roles. Interviews and focus groups were audio-recorded and transcribed verbatim by a professional transcribing service. Written, informed consent was obtained from all participants.

\section{Data analysis}

Transcripts were verified against audio recordings by one investigator (ET). Data management was facilitated using NVivo V.9.0 (QSR, Melbourne). Interview transcripts, recordings, narrative reports and field notes were entered into the software. All data were collected, entered and then analysed together. Two investigators 
(ET and KS) read the transcripts and independently analysed the data inductively, coding the data for emergent themes. ${ }^{19}$ The initial coding and emerging themes were then discussed between ET and KS to reach a general consensus. Results were then presented at meetings involving all authors, where discrepancies were resolved and themes finalised. Following thematic analysis, theoretical frameworks were used to explain the findings. Illustrative quotes that represent a range of stakeholders and points of view were selected for reporting.

\section{RESULTS}

\section{Participant characteristics}

Eighteen individual interviews were conducted with patients, 11 of whom were women. The mean age of participants was 72.6 years (range 52-85 years), the median number of medicines was 11 (range 6-16) and the median number of medication-related problems identified by the practice pharmacist was 2 (range $0-8$ ). Participants had a range of chronic medical conditions (eg, asthma, depression, diabetes, hypertension and osteoporosis). Twelve patients were recruited from the private practice and six from the community health centre. This corresponded to roughly $20 \%$ of participants from each site.

Practice staff focus group 1 had six participants (5 GPs, 1 practice nurse) and focus group 2 had eight (4 GPs, 3 practice nurses, 1 practice manager). Of the practice staff participants, eight were women, the mean age was 49.4 years (range 37-64) and the median duration of general practice work experience was 27 years (range 3-33). All practice staff who had worked with the practice pharmacist and were working on the day of the focus group participated. One practice manager from the private practice was unavailable, while a nurse and GP from the community health centre were not available. No staff refused to participate.

The two practice pharmacists had at least 8 years of experience undertaking home medicines reviews. ${ }^{20}$

\section{Major themes}

Five major themes that illustrate the experiences of the participants emerged: environment; professional relationships and integration; pharmacist attributes; staff and patient benefits and logistical challenges.

\section{Environment}

Patients felt comfortable seeing the pharmacist in the clinic and appreciated the privacy in consulting rooms. By being affiliated and present within the clinic, rapport and trust with the pharmacist were more easily built.

I could have asked the pharmacist that I usually see exactly the same questions but you actually don't ever get the chance to have that two way dialogue with that pharmacist [in the community pharmacy] and the other thing was, in the clinic you were in a private room,... which meant that we could sort of chat. (patient 7)
I think people are comfortable seeing other health professionals here. I think they know the clinic, they feel comfortable here. It's not invading their home if they're not keen for that. (GP5)

I think there's a greater acceptance from the client because the pharmacist is part of the team. (practice manager 1)

I've found the patients have a different mindset when attending a consultation at the clinic. They seem to approach the service with a greater degree of respect and appreciation. I believe this is due to the professional environment and a more tangible association with their GP. (pharmacist 1)

Pharmacists enjoyed working with a diverse mix of staff in the clinic and perceived delivery of services in the clinic environment as more clinical and professional in nature than services provided outside the clinic (eg, within a community pharmacy).

I loved having... access to a lot of different health professionals. There was the dietician that I often had discussions with and physiotherapists. The nursing staff certainly, and obviously the medical staff. So it was great to be able to have that professional interaction with a variety of different health professionals. (pharmacist 2)

The presence of the pharmacist within the clinic improved access to medicines information and enabled verbal communication about medication-related issues (rather than written communication that typically occurs between GPs and pharmacists with the Australian home medicines review programme). ${ }^{20}$ It encouraged medication issues to be discussed and resolved in a timely manner and facilitated referrals to the pharmacist. This ease of communication also aided in the development of rapport.

Having someone on site even just to ask quick questions to -I thought the pharmacist was really helpful on occasion. Obviously they have an overall idea of medications...so it was nice to have [the pharmacist] there. (GP4)

You always work better with people when you eyeball them. (nurse 1)

Patient-specific conversations [with GPs] often take place before [patient] consultation and problem areas identified to be focused on. (pharmacist 1, narrative report 1 month)

Certainly on a one-to-one communication verbally you do have greater chance of explaining why you are making certain recommendations [to the GP], and you can justify it and then have that discussion about...the recommendations. (pharmacist 2)

We'd bump into each other in the tea room or in the hallway-so there weren't any barriers to communication [with practice staff] given that we were under the same roof and that's the major advantage. (pharmacist 1 ) 


\section{Integration and professional relationships}

Pharmacists mostly had positive experiences with integrating into the primary care practices. Positive experiences were facilitated by supportive staff who already worked within established interdisciplinary teams and, in the case of one pharmacist, a previous working relationship with some GPs in the clinic (as a result of having conducted home medicines reviews for the clinic).

I had a pre-existing relationship with a couple of the GPs there, which certainly was a factor, but just basically their culture. They have a psychologist,... a diabetes educator, ...pathology, so they already had experience in incorporating other disciplines-so I think that helped as well. ... So I think it was a combination of those factors and that they also saw value in what we were doing, which is a very important part of them embracing it. (pharmacist 1)

Initial challenges faced by pharmacists included some staff lacking experience of working with pharmacists previously and thus not being familiar with the expertise or role of a non-dispensing pharmacist and therefore not utilising them fully.

Each staff member is very different...Some of them were a little bit more resistant than others. (pharmacist 2)

To overcome cultural and professional barriers to integration, pharmacists had to be flexible, familiarise themselves with individual staff members and learn how to complement their roles.

I think it was really important to get to know each staff member and what their agenda....and what their needs were. To see how I could support them in what they were trying to do. (pharmacist 2)

These initial challenges to integration generally dissipated once the pharmacist was used to the practice environment and staff became familiar working with a pharmacist. Pharmacists felt that this would continue to develop over time.

The nursing staff were quite quick to embrace the expertise of the pharmacist whilst the GPs took a little more time, not really being sure at the outset of what the clinic pharmacist's skills and/or knowledge would be. The role developed over time and I believe started to hit its straps as the allocated time came to an end, with the GPs becoming more used to using me as an information source. (pharmacist 2, narrative report 6 months)

The integration of pharmacists into the practice allowed rapport to be built between the pharmacist and other staff. Staff were able to learn about the pharmacist's role and started to view them as a team member. Pharmacists also enjoyed working within a team environment.

You just see the pharmacist more as part of the team rather than someone who dispenses the script...you have got a bit more collegiality, and more bouncing things off one another. A bit more interactive and educative for the both of us. (GP6)

I loved being part of a team. (pharmacist 2)

The practice pharmacists also developed good relationships with the local community pharmacies. This facilitated continuity of care and was appreciated by the clinic staff.

They were great, and very, very supportive...the pharmacies around the place. I didn't have ever one pharmacy refuse to send a dispensing history or to discuss a person's medication. They were quite comfortable with that. It's good to keep them in the loop. (pharmacist 2)

[The practice pharmacist] usually followed up with the [community] pharmacists as well. Sort all that out too with the local pharmacist...It was brilliant. (practice manager 1)

The relationship between patients and practice pharmacists were deemed to be positive. Patients appreciated being able to spend time with the practice pharmacist specifically discussing their medications, compared with their GP or community pharmacist who were often viewed as being too busy. Patients also felt that the practice pharmacist would not adversely affect their relationship with the GP and might improve interprofessional communication and relationships.

You go to the doctor and they're pressed for time always and they'll explain things to you but not in such detail as what the pharmacist did. (patient 1)

Because if you are in the busy pharmacy, it's very difficult for the pharmacist and for you and usually there's no time to ask. (patient 18)

[The pharmacist] had more time to spend with them one-on-one especially with respect to their medications. So I found patients get confused with their medications quite easily and I guess they tell you they are taking them fine, but when you really press them they're not. I guess I don't have time to really press them... (GP4)

Although patients were generally receptive to seeing the practice pharmacist, there were some challenges. These included patients being initially confused about the purpose of seeing the practice pharmacist or being reluctant to attend yet another appointment with a health professional. Some patients preferred consulting their GP rather than talking to the practice pharmacist about their medicines.

I have also identified varying attitudes towards the service. Most patients have embraced it wholeheartedly, whilst others have felt inconvenienced and arrive with an attitude of 'this is pointless' or 'I am doing this as a favour for my GP who is a good bloke'. (pharmacist 1, narrative report 1 month) 
The doctor just told me 'I'm going to do an appointment for you to see the pharmacist' and that's it and I haven't any idea what's going on, just they told me 'bring all your tablets with you'. (patient 11)

Once you've got a doctor tell you what to do and then prescribe your tablets, if something is not working you're talking to your doctor, you're not talking to your pharmacist. The pharmacist ...what can he do? He can only say get a blood test...Your doctor-he's the best man you can get. (patient 17)

I think it's a good thing to have...every so often it's a good thing to be able to sit down and go through things with a pharmacist. ( patient 9)

It was felt by the pharmacists that these challenges could be overcome with adequate promotion of pharmacist services. Additionally, most negative receptivity disappeared once patients experienced the benefits of the practice pharmacist services.

I didn't really have a lot of resistance. There was just that comment that 'I don't know why I am here. I know what I am doing with my medicines'. But usually at the end of the consultation they were very positive to say 'Oh I actually did learn something about my medicines'. (pharmacist 2)

\section{Pharmacist attributes}

Participants felt that the success of pharmacist integration into the clinic setting was influenced by the pharmacist's personality, skills and attributes. In particular, practice pharmacists needed to be personable, flexible and have sound interpersonal and communication skills.

[The practice pharmacist] was very, very patient and she gave the impression she really knew what she was talking about...she could explain everything. (patient 18)

She was very good, assertive with the GPs, but very gentle with the clients I thought. From day one, she had authority, she had that sort of presence, so it sort of made you respect her. (GP4)

...you also need to work in a team environment so there's a certain type of person that can do that. You need to be good with people obviously, because you're consulting one-on-one, so a 'people person' as well, so there is a particular personality type that would be best suited to the role. (pharmacist 1)

I think you have to go in there with an open mind and look at the needs of the clinic rather than going in with your fixed ideas...Be flexible, be open-minded... (pharmacist 2)

The success of the role also relied on the pharmacist being proactive and actively engaging with staff and identifying potential clients.
She was good because she didn't let any opportunities slip by. If there was a discussion that involved medication of clients, her ears would prick up and then she'd get involved appropriately. (nurse 1 , mental health)

The role requires a proactive pharmacist who takes an active role in seeking out relationships with GPs, nursing staff, allied health professionals and the admin staff - all crucial in the success of the position. (pharmacist 2, narrative report 6 months)

\section{Staff and patient benefits}

Staff benefited from sharing patient care with the pharmacist; pharmacists could offer reassurance and feedback to staff, especially as they had time to explore medication issues in depth.

I used to refer to [practice pharmacist] if a patient didn't need to see a doctor because they didn't need a script but over-the-counter medication... because there wasn't a doctor available for a consult. (nurse 2)

I thought it was a logical conclusion to have someone who has that view of therapeutics. We are all competent at prescribing and considering interactions...But it's... helpful to have a second opinion, a second pair of eyes, because we don't have a mortgage on knowledge. (GP7)

[The practice pharmacist] actually had the time, did a lot of research and ringing the pharmacy down the road and doing the home visits, so it really helped with compliance because...we don't always have the time, she took it to another level I guess than what I would normally do. (nurse 1)

Staff also benefited from an increased awareness of the actual medicine taking practices of their patients. The findings from the consultations also encouraged GPs to not only consider the pharmacist's recommendations, but also think of other patient issues and update their records.

I think it would definitely increase the GP's knowledge of what their patients were doing. Because it was very, very rare for the GP's list of medications to reconcile with what the patient was actually taking. So if nothing else, at least I am feeding back to the GP this is what you have prescribed but actually this is what they are taking. (pharmacist 2)

Oh it was great, and then to go back to the doctor's to get the feedback from what the pharmacist and I had talked about. The doctor didn't know that I didn't know that about my medication, it sort of hadn't arisen before, so it was actually a really good two way street. (patient 8)

I think it also makes you update your [patient's] health summary...in a way that it's very clear as to what the patient has got, therefore why they are on the treatment. (GP5) 
The practice pharmacist also assisted staff with improving the quality of prescribing and medicines information and management within the clinic.

I think one of the good things...is that it's worked...for the clinicians, and I think they're spending a heap more time on their medications now. (practice manager 1)

Participants felt that the pharmacist improved the patients' understanding and awareness of their medicines, provided reassurance, encouraged compliance, rationalised drug therapy and optimised health outcomes.

It was a benefit...it makes you feel like you are doing the right thing...making sure you're up with the tablets. (patient 14)

Just having somebody to go over the medications and discuss [them] with the patients and explain to them what they were for, and actually confirm that they were actually taking the stuff, checking their compliance etc. (GP1)

[The appointment with the pharmacist was] very informative. It probably helped me understand the medication more clear [sic] than I had in the past because I knew that I was taking medication for certain things, but I probably had a couple of the tablets mixed up. (patient 7)

Being within the clinic and part of the healthcare team, the pharmacist also began to see the patient more holistically and became involved in the patient's overall management plan.

I found that I had more contribution to the overall management of a patient rather than just their medicines. So their social circumstances impacted on their medication management but sometimes their social circumstances were more of an immediate issue that needed to be addressed. (pharmacist 2)

\section{Logistical challenges}

While the presence of the pharmacists within the clinics was well received, the logistics of accommodating them, including office space, posed an issue.

My shifts are often divided between different rooms. (pharmacist 1)

There were problems when [the pharmacist] couldn't get a room. She didn't always have a room to work in. (practice manager 1)

Time was an issue for practice staff and patients. Staff were busy with their day-to-day routine and did not always have time to engage fully with the pharmacist.

[The pharmacist] was fantastic with the patients she saw, but I just felt guilty the whole time she was here because
I actually didn't have time to really access her or refer people to her. (GP2)

The biggest challenge is modifying the GPs' behaviours. All of the services need to be driven by the GPs. They are very time poor and can be in automatic pilot mode. Support staff and software programs can assist the identification of eligible patients, but the onus still remains with the GP at the point of consultation. (pharmacist 1)

Some patients found attending appointments burdensome.

...just another one of the millions of other appointments I have regarding what's going on with me at the moment. (patient 3)

Pharmacists were only available for a limited number of hours each week and on particular days, which was viewed as a disadvantage. They found it challenging to manage their time within busy practices. Their workload would often fluctuate from week to week.

My greatest challenge...time! (pharmacist 2, narrative report 2 months)

Well [the pharmacist] has got a fixed day and times, as opposed to after hours or multiple days to pick from. (GP5)

There were often days that she wasn't really busy at all. (GP4)

...but she might not be there on the day that you need them. (patient 10)

The difficulty of course is that I was only there once a week...each week you have to regenerate that role that you have and that presence that you have. (pharmacist 2)

The findings from this qualitative evaluation of colocation of pharmacists in general practice may be explained using two theoretical frameworks that describe interprofessional collaboration and the adoption of new services. The structuration model of interprofessional collaboration, ${ }^{21}$ which has its basis in organisational sociology, consists of four interrelated dimensions including: shared goals and vision; internalisation; formalisation and governance. The dimension of shared goals and vision was attained by the pharmacists' and practices' common desire to provide optimal patient care through improved medication management. The dimension of internalisation, characterised by mutual acquaintanceship and trust, was exemplified by the processes of pharmacist integration and relationship building with staff and patients. Once the staff became familiar with the pharmacists' skills, trust was built and a sense of team established. The dimension of formalisation, which encapsulates the structuring of clinical care, was highlighted in the way the pharmacist's role was centred on medicines management, allowing staff to share roles 
and patient care. Information exchange also occurred easily given the interdisciplinary environment. The dimension of governance, which involves the leadership functions that support collaboration, was achieved by appropriate guidance from practice managers and head GPs who provided support for innovation and teamwork. Logistical issues, especially limited time for interprofessional interaction, posed an underlying barrier (see diagram in online supplementary file 5).

According to Rogers's 'diffusion of innovation theory', 22 the adoption and diffusion of an innovation is determined by five characteristics: relative advantage, compatibility, complexity, trialability and observability. Relative advantage-the degree to which an innovation is perceived to be better than what it supersedes-is exemplified in our study by the improved positive outcomes and opportunities for interprofessional communication and collaboration compared to existing services. The practice pharmacist services displayed compatibility with the practices' existing values of client-focused and team-based care, and built on staff's previous positive experiences with consultant pharmacists. The complexity of the intervention-particularly the study processes for identification and referral of patients to the pharmacist-was minimised by having pharmacists who were proactive and adaptable to suit the needs of individual staff members. The pharmacy service was successfully trialled and the results of the innovation were observable by patients and staff through the pharmacists' contribution to quality use of medicines.

\section{DISCUSSION}

This study explored the perspectives of patients, staff and practice pharmacists on the role of pharmacists colocated within the Australian general practice setting. Participants reported that colocation and the interdisciplinary environment of general practice enabled better communication and collaboration compared to traditional pharmacy services. Participants felt that pharmacists needed to possess certain attributes to ensure successful integration. Pharmacist services were perceived to provide benefits for patients and staff; however, attitudinal, professional and logistical challenges posed barriers. Application of D'Amour's structuration model of interprofessional collaboration and Rogers's diffusion of an innovation theory helped to explain our findings and the successful integration of the practice pharmacist into the interdisciplinary primary care team.

This study has strengths and limitations. Its strengths were that it involved two types of general practice clinic -one private practice and one community health centre-and used a combination of qualitative methods. The study's limitations were that it involved a small number of clinics, which had established interdisciplinary teams and were receptive to adding a pharmacist to their team, so it may not be representative of all general practice clinics. Additionally, the pharmacists were experienced and, in one case, had a previous working relationship with the practice (not colocated). Study constraints, including short duration and limited pharmacist hours, were also limitations.

Other studies have explored stakeholder views on pharmacist integration into co-located primary healthcare teams. Pottie et $a l^{12}$ explored Canadian physicians' perceptions of pharmacist integration through focus groups and interviews. While physicians reported similar benefits and concerns to those identified in our study, the issues of security and medicolegality were elucidated only in their study. This may be because many Australian GPs, including those in our study, had experience of working with consultant pharmacists and were comfortable that the practice pharmacist would not cross ethical and legal boundaries. Consultant pharmacists are independent pharmacists accredited to undertake medicines reviews, but are not co-located in GP clinics. Canadian pharmacist narratives 818 revealed similar concerns in the early stages of integration, which gradually diminished with time. Similar to our study, other studies have found that colocation, existing working relationships and trust development were important factors for pharmacist integration. ${ }^{23-25}$ Petty et $a l^{13}$ explored patients' views of pharmacist-conducted medication review clinics within a general practice surgery in the UK. Similar to our study, patients had a range of positive and negative views before and after seeing the practice pharmacist.

Previous Australian studies ${ }^{14} 15$ on this topic generally have not involved participants who have experienced a practice pharmacist. Those studies suggested various potential benefits of colocation, such as patient privacy, improved access to patient information and increased interprofessional rapport and communication, and these perceived benefits were confirmed in our study. ${ }^{14}$ Additionally, some proposed desirable pharmacist attributes and logistical challenges raised in the previous studies aligned with our findings. ${ }^{14}$ Compared with other studies, the practice pharmacists in this study highlighted some additional benefits of working in this role. These included the ability to work with a diverse range of staff, including nursing and allied health, emphasising the interdisciplinary nature of the role; that interprofessional communication could occur prior to consultations, resulting in improved delivery of services; and the way the pharmacists now viewed patients more holistically and felt integrated into their overall management. While previous studies found that some participants were concerned about the potential negative effect that the practice pharmacist role would have on relationships with GPs and the role of community pharmacists, we did not observe this. ${ }^{14}$

This study highlights various barriers and facilitators that need to be considered by practitioners and policymakers when integrating a pharmacist into the primary healthcare team. Integration is facilitated by colocation, communication and positive pharmacist characteristics, including credibility, adaptability and proactivity. Supportive staff, shared goals and the creation of 
benefits for patients and staff are imperative. Logistical issues, especially time and office space, are barriers to be considered. Future research should investigate the feasibility, sustainability and financial viability of general practice pharmacist roles and evaluate the impact on patient outcomes in larger controlled studies.

\section{CONCLUSION}

To our knowledge, this is the first qualitative study to explore the experiences of general practice staff, practice pharmacists and patients on their interactions within the Australian general practice environment. Overall, participants were receptive of colocated pharmacist services. The interdisciplinary environment enabled interprofessional collaboration. Integration and relationship formation developed over time. Patients and staff benefited from these services; however, logistical challenges posed a barrier.

Acknowledgements We would like to thank the participants of this study for their time and contributions.

Contributors ET contributed to the study concept and design; acquisition, analysis and interpretation of data; drafting and critical revision of the manuscript. KS, RE and JG contributed to the study concept and design, analysis and interpretation of data, as well as the critical revision of the manuscript.. All the authors have approved the manuscript.

Funding This research received no specific grant from any funding agency in the public, commercial or not-for-profit sectors.

Competing interests None.

Ethics approval Monash University Human Research Ethics Committee.

Provenance and peer review Not commissioned; externally peer reviewed.

Data sharing statement No additional data are available.

Open Access This is an Open Access article distributed in accordance with the Creative Commons Attribution Non Commercial (CC BY-NC 3.0) license, which permits others to distribute, remix, adapt, build upon this work noncommercially, and license their derivative works on different terms, provided the original work is properly cited and the use is non-commercial. See: http:// creativecommons.org/licenses/by-nc/3.0/

\section{REFERENCES}

1. Fish A, Watson M, Bond C. Practice-based pharmaceutical services: a systematic review. Int J Pharm Pract 2002;10:225-33.

2. Borenstein JE, Graber G, Saltiel E, et al. Physician-pharmacist comanagement of hypertension: a randomized, comparative trial. Pharmacotherapy 2003:23:209-16.

3. Dolovich L, Pottie K, Kaczorowski J, et al. Integrating family medicine and pharmacy to advance primary care therapeutics. Clin Pharmacol Ther 2008;83:913-17.
4. Zermansky AG, Petty DR, Raynor DK, et al. Randomised controlled trial of clinical medication review by a pharmacist of elderly patients receiving repeat prescriptions in general practice. BMJ 2001;6:1340-3.

5. Yates R, Wells L, Carnell K. General practice based multidisciplinary care teams in Australia: still some unanswered questions. A discussion paper from the Australian general practice network. Aust J Prim Health 2007;13:10-17.

6. Ackermann E, Williams I, Freeman C. Pharmacists in general practice - a proposed role in the multidisciplinary team. Aust Fam Physician 2010;39:163-4.

7. Australian Council for Safety and Quality in Healthcare. Second national report on patient safety-improving medication safety. Canberra: Australian Council for Safety and Quality in Health Care, 2002.

8. Farrell B, Pottie K, Haydt S, et al. Integrating into family practice: the experiences of pharmacists in Ontario, Canada. Int J Pharm Pract 2008;16:309-15.

9. Farrell B, Pottie K, Woodend K, et al. Shifts in expectations: evaluating physicians' perceptions as pharmacists become integrated into family practice. J Interprof Care 2010;24:80-9.

10. Kolodziejak L, Rémillard A, Neubauer S. Integration of a primary healthcare pharmacist. J Interprof Care 2010;24:274-84.

11. MacRae F, Lowrie R, MacLaren A, et al. Pharmacist-led medication review clinics in general practice: the views of greater Glasgow GPs. Int J Pharm Pract 2003;11:199-208.

12. Pottie K, Farrell B, Haydt S, et al. Integrating pharmacists into family practice teams: physicians' perspectives on collaborative care. Can Fam Physician 2008;54:1714-17.

13. Petty D, Knapp P, Raynor D, et al. Patients' views of a pharmacist-run medication review clinic in general practice. $\mathrm{Br} J$ Gen Pract 2003;53:607-13.

14. Tan E, Stewart K, Elliott RA, et al. Integration of pharmacists into general practice clinics in Australia: the views of general practitioners and pharmacists. Int $J$ Pharm Pract. Published Online First: 12 June 2013. doi:10.1111/ijpp.12047

15. Freeman C, Cottrell W, Kyle G, et al. Pharmacists', general practitioners' and consumers' views on integrating pharmacists into general practice. J Pharm Pract Res 2012;42:184-8.

16. Freeman C, Cottrell W, Kyle G, et al. Integrating a pharmacist into the general practice environment: opinions of pharmacist's, general practitioner's, health care consumer's, and practice manager's. BMC Health Serv Res 2012;12:229.

17. Tan E, Stewart K, Elliott RA, et al. An exploration of the role of pharmacists within general practice clinics: the protocol for the pharmacists in practice study (PIPS). BMC Health Serv Res 2012;12:246.

18. Pottie K, Haydt S, Farrell B, et al. Narrative reports to monitor and evaluate the integration of pharmacists into family practice settings. Ann Fam Med 2008;6:161-5.

19. Liamputtong P. Qualitative data analysis: conceptual and practical considerations. Health Promot J Austr 2009;20:133-9.

20. Medicare Australia. Home medicines review. 2012. http://www. medicareaustralia.gov.au/provider/pbs/fourth-agreement/hmr.jsp

21. D'Amour D, Goulet L, Labadie J-F, et al. A model and typology of collaboration between professionals in healthcare organizations. BMC Health Serv Res 2008;8:188.

22. Rogers E. Diffusion of innovations. New York: Free Press, 1983

23. Jesson JK, Wilson KA. One-stop health centres: what colocation means for pharmacy. Health Place 2003;9:253-61.

24. Zillich A, McDonough RP, Carter BL, et al. Influential characteristics of physician/pharmacist collaborative relationships. Ann Pharmacother 2004:38:764-70.

25. Bradley F, Elvey R, Ashcroft DM, et al. The challenge of integrating community pharmacists into the primary health care team: a case study of local pharmaceutical services (LPS) pilots and interprofessional collaboration. J Interprof Care 2008;22:387-98. 